\title{
Plate-impact loading of cellular structures formed by selective laser melting
}

\author{
R. E. Winter ${ }^{1}$, M. Cotton ${ }^{1}$, E. J. Harris ${ }^{1}$, D. J. Chapman ${ }^{2}$, \\ D. Eakins ${ }^{2} \&$ G. McShane \\ ${ }^{1}$ AWE, Aldermaston, Reading, Berkshire, UK \\ ${ }^{2}$ Institute of Shock Physics, Imperial College, London, UK \\ ${ }^{3}$ Department of Engineering, University of Cambridge, UK
}

\begin{abstract}
Studies of the shock loading of porous material have the potential to improve our understanding of factors such as density, crush strength and pore size on energy absorbing capability. Porous components were manufactured using Selective Laser Melting (SLM) in which layers of metal powder are fused together to create a structure specified by an electronic file. Samples have been manufactured in which a lattice is formed by an array of intersecting rods angled at 45 degrees to the surface of a $6 \mathrm{~mm}$ thick $\mathrm{x} \sim 100 \mathrm{~mm}$ diameter disc. The cell size is $1 \mathrm{~mm}^{3}$ and the density is $44.6 \%$ of solid. A $100 \mathrm{~mm}$ gas gun has been used to impact the porous samples onto solid stainless steel plates. Het-V laser interferometry was used to measure the velocity vs. time profile of the transmitted shock. The experimental results were compared with three dimensional computer predictions. It was found that the simulations reproduced the main features of the experimental record but tended to underestimate the measured velocities, suggesting that the codes were not calculating the energy absorbed by the lattice correctly. Additional calculations were performed with the aim of building a picture of the processes of energy absorption in cellular materials whose structure is varied systematically. These supporting studies suggest a possible explanation for the observed computational/experimental discrepancies.

Keywords: cellular structures, truss panels, selective laser melting, additive manufacture, impact, shock.
\end{abstract}




\section{Introduction}

Selective Laser Melting (SLM) is an additive manufacturing technique which allows cellular materials, which have the potential to form structures to protect against fragment and blast loading, to be manufactured according to a specification encoded in an electronic file. This process offers the ability to produce complex internal geometries with features on the scale of hundreds of microns. Further, in principle, it allows structures to be tailored to provide optimal protection against specific threats and has the potential to improve our understanding of the effects of factors such as density, and cellular structure and scale, on energy-absorbing capability.

SLM has been used to investigate designs utilising a range of structures based on intersecting struts. For example Mines et al. [1] and Deshpande and Fleck [2] have investigated body-centred-cubic and tetragonal structures respectively. Wang et al. [3] have studied a structure designated "Kagome" which has a threefold axis of symmetry and McKown et al. [4] have studied an octahedral lattice.

AIn principle, the relative merits of alternative cellular configurations can be assessed by computer simulation. However, a prerequisite of such simulations is to determine the validity of the chosen code in the relevant loading regime. The Sandia National Laboratories code, CTH [5] has a 3D capability, together with the ability to read in structures in the form of surface triangle lithography (stl) files. Since the SLM process is controlled by an stl file it follows that the SLM structures can be fed in to $\mathrm{CTH}$, thereby allowing 3D simulations to be run.

In the work described in this paper the results of plate impact experiments on a trial stainless steel cellular structure are compared with CTH simulations. It was found that the simulations gave a good qualitative agreement with the experiments but that there were quantitative differences between the simulations and the experimental data. The code/experimental study led to an extended study of a simplified cell. The aims of the computer analysis were to provide a picture of the processes in a deforming cell, determine the effect of factors such as impact velocity and cell configuration on energy absorption and, hopefully, to shed light on the code experimental discrepancies observed in the initial, MS1, study.

\section{CTH versus experiment}

The structure and material chosen for our initial study was loosely based on the configuration studied by McKown et al. [4] who used SLM to manufacture structures consisting of intersecting rods (or strands) of $316 \mathrm{~L}$ stainless steel. The MS1 structure on which our study is based, is shown in fig. 1, and is significantly more dense than those studied by McKown. The reason is that an important aim of the work was to compare the experimental results with the predictions of 3D CTH simulations. The maximum mesh size it is reasonable to use is determined, to a large extent, by the smallest features in the structure. In other words narrow struts require finer meshes. This latter consideration led us to choose a strut diameter $(0.4 \mathrm{~mm})$ which was an appreciable fraction of the cell 
size $(1 \mathrm{~mm})$. As shown in fig. 1 the structure chosen consists of an array of $316 \mathrm{~L}$ stainless steel vertical columns of diameter $0.4 \mathrm{~mm}$ separated in a square array by $1 \mathrm{~mm}$; the column structure is intersected by rods angled at 45 degrees as shown. The structure was estimated to be $44.6 \%$ void by volume giving a relative density (as defined in [4]) of 1.805 .


Figure 1: The structure chosen for experimental testing (designated MS1).

A series of 3D CTH calculations were run to assess the relative merits of different experimental configurations. The mesh size was $50 \mu \mathrm{m}$. The hydrodynamic compressibility was modelled using a Mie-Gruneisen equation of state and strength was modelled using the Steinberg-Guinan strength model [6].

According to $\mathrm{CTH}$, the wave delivered to the solid target by the impact was initially structured across the impact face, but had smoothed out significantly by the time it reached the back face. Therefore the velocity computed at the back face was not unduly sensitive to position across the face. Following the computer study it was decided to conduct a series of experiments based on the set-up shown in fig. 2. The cellular component is in the form of a $99.6 \mathrm{~mm}$ diameter $\mathrm{x}$ $6 \mathrm{~mm}$ thick disc. The vertical rods in fig. 1 are aligned parallel to the axis of the sample disc. Since each cell occupies a $1 \mathrm{~mm}$ cube there are 6 cells through the thickness of the disc. The disc was protected at its periphery by a $1 \mathrm{~mm}$ thick $\mathrm{x}$ $6 \mathrm{~mm}$ long cylinder formed by the SLM process. Therefore there were about 100 cells across a diameter. The diagnostics consisted of a ring of 6 ionisation probes
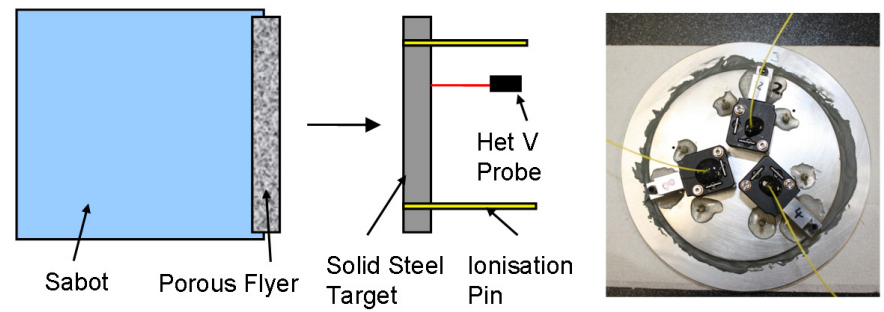

Figure 2: Experimental set-up and photograph showing the position of the three velocity probes. The experiments were conducted on the $100 \mathrm{~mm}$ gas gun at The Institute of Shock Physics, Imperial College, London. 
to determine impact time and tilt and 3 "Het-V" velocity probes [7] arranged as shown in the photograph in fig. 2.

The measurements obtained in experiments conducted with nominal flyer velocities of $300 \mathrm{~m} / \mathrm{s}, 500 \mathrm{~m} / \mathrm{s}$ and $700 \mathrm{~m} / \mathrm{s}$ are shown for comparison with the corresponding $\mathrm{CTH}$ calculations in fig. 3. It is seen that, at each velocity, the differences between the three experimental velocity records are small. Comparison with the code provides a explanation of the features seen in the experimental traces. In all of the traces an elastic precursor of amplitude $\sim 25 \mathrm{~m} / \mathrm{s}$, generated by the initial contact between flyer and target is seen. The collapse of the porous flyer to solid, or near solid, density then generates a steep rise to a plateau. After $\sim 2 \mu$ s the velocity traces rise to a second plateau as the wave generated at the back face of the target reflects of the impact interface then reaches the back face again. Superimposed on all of the traces are the oscillations generated by the successive collapse of the cells.

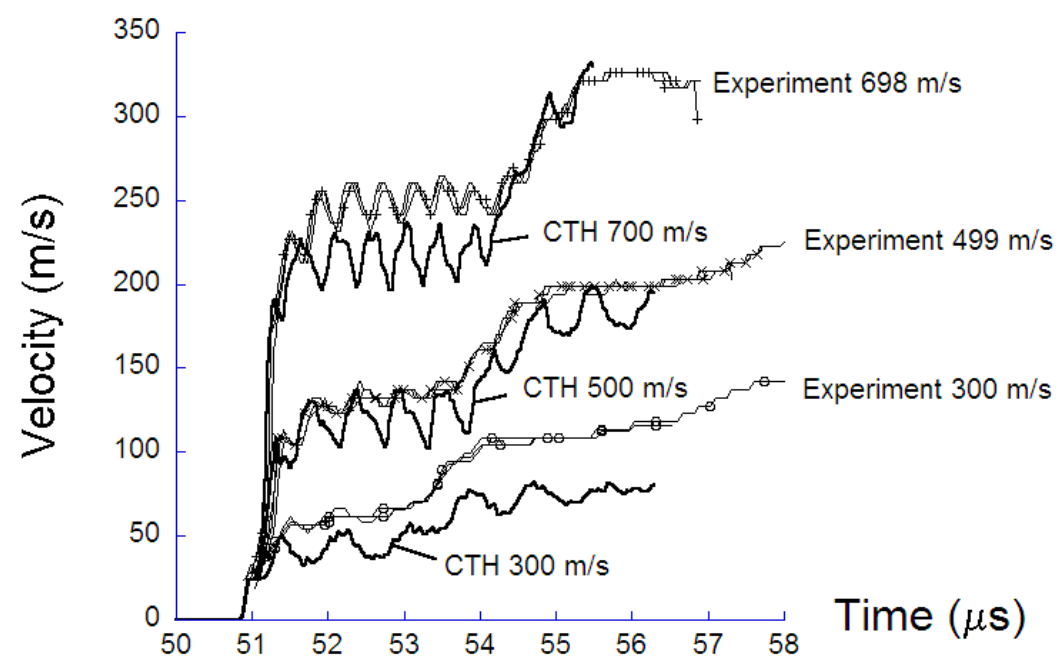

Figure 3: Experiment versus CTH simulations.

Although, encouragingly, there is qualitative agreement between the calculations and experiment, there are some quantitative differences. The amplitude of the oscillations is greater in the simulations than in the experiments suggesting that at the time and distance scale of the cells, the perturbations in the velocity vs. distance field are greater in the simulations than occurs in reality. Another significant difference is that the code tends to underestimate the particle velocity transmitted to the target, suggesting that the energy absorbed by the cellular material is greater in the simulation than in the experiment.

We concluded from our observations that a systematic CTH study of energy absorption by cellular structures should be conducted. The aims were to shed light on the probable reasons for the discrepancies between simulation and 
experiment and at the same time to build an understanding of the factors controlling the absorption of impact energy by cellular structures.

\section{CTH study of energy absorbing mechanisms}

Figure 4 shows a simplified two dimensional configuration, chosen as a basis for a systematic study. The energy absorbing element (EAE), shown by the grey square, consists of a $6 \mathrm{~mm}$ square extending into the paper. The hatched rectangle illustrates the orientation of the "rod" variant (see fig. 5). The flyer and the target are rectangles of dimensions $6 \mathrm{~mm} \times 18 \mathrm{~mm}$, again extending into the paper. CTH was used to generate time sequences showing materials, plastic flow, internal and kinetic energy, pressure, temperature and flow stress. The total kinetic and total internal energy of the flyer, EAE and target were also output from $\mathrm{CTH}$.

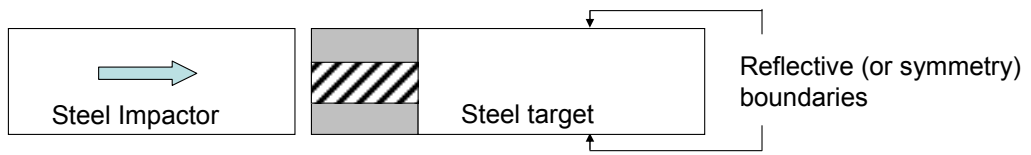

Figure 4: Schematic of the 2D configuration used a basis for CTH study of energy absorption. Simulations were run with a range of structures in the region shown as a grey square.

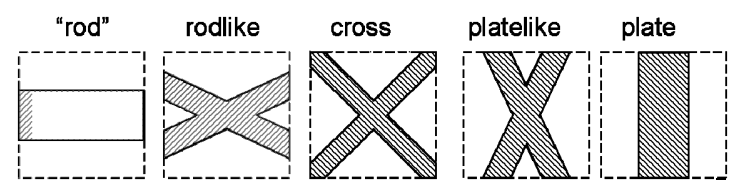

Figure 5: The five structures which were assessed using CTH. The impactor approaches the cell from the left and the target is on the right.

The MS1 structure, for which results were presented earlier, consists of a mixture of rods at various angles to the impact direction. It is possible that the codes are better at simulating the response of some types of elements than others. Therefore we decided to run simulations of the five alternative EAE structures depicted in fig. 5. In each case the flyer impacts from the left and, as depicted in fig. 4, the target, or acceptor, is on the right. For convenience the EAE on the extreme left is labelled "rod". However, since this is a 2D setup the element really consists of a plate extending into the paper. The structure on the extreme right also consists of a plate extending into the paper but, it is rotated by $90^{\circ}$ relative to the structure on the left. The structure in the centre, labelled "cross" consists of two plates at 90 degrees to each other. The inclusion of the rod-like and plate-like structures provides a transition from rod to plate structure.

The sequence in fig. 6 plots internal energy for a rod-like cell impacted at $900 \mathrm{~m} / \mathrm{s}$. Full densification of the cell occurs at $\sim 4.8 \mu \mathrm{s}$ after which time there is very little change in the internal energy distribution in the vicinity of the EAE. 
Note that intense flow occurs at the edges of the cell as mushrooming of the angled plates produces jets which eventually impinge on the end-face of the target. The highest specific energies seen in the graphics are generated as a result of this jet impact.

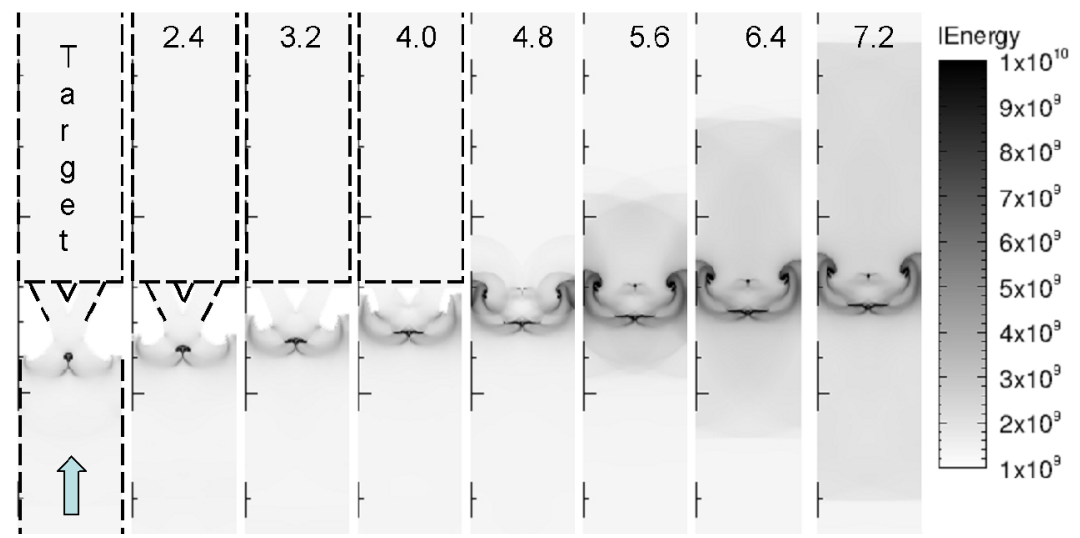

Figure 6: A CTH sequence showing internal energy generation in a rod-like structure impacted (from below) at $900 \mathrm{~m} / \mathrm{s}$. The numbers at the top are time in $\mu \mathrm{s}$. For clarification some of the components are delineated by dashed lines. The units are ergs/gm.

As expected the different EAEs respond differently. Figure 7 shows internal energy, plastic strain and temperature plots for the five EAE variants just after

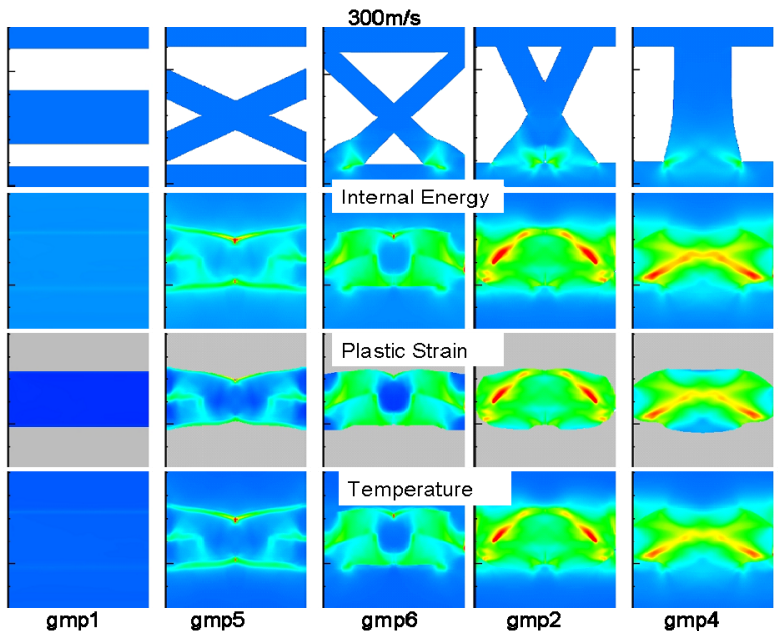

Figure 7: The effect of cell geometry on plastic strain, internal energy and temperature. Internal energy is shown at both early and late times. plastic strain and temperature are shown at late times only. 
full densification for a simulation at $300 \mathrm{~m} / \mathrm{s}$. It is seen that the energy absorbed by the EAE tends to increase as the configuration is varied from plate to rod but that there is little difference between the rod-like and rod variants. At this relatively low velocity none of the variants exhibit pronounced mushrooming or jetting.

A useful comparison of the effectiveness of the different structures as energy absorbers is provided by plotting the energy reduction of the impactor and the energy increase of the target. On the left hand side of fig. 8 Internal Energy (IE) and Total Energy (Internal + Kinetic) are plotted against time for the impactor. As expected the initial total energy of the flyer is reduced more by a rod or rodlike EAE than by a plate or plate-like EAE. Similarly on the right of fig. 8 it is seen that, although there are some variations with time, more energy tends to be delivered to the target with a plate and plate-like EAE than with rod-like or rod EAE.
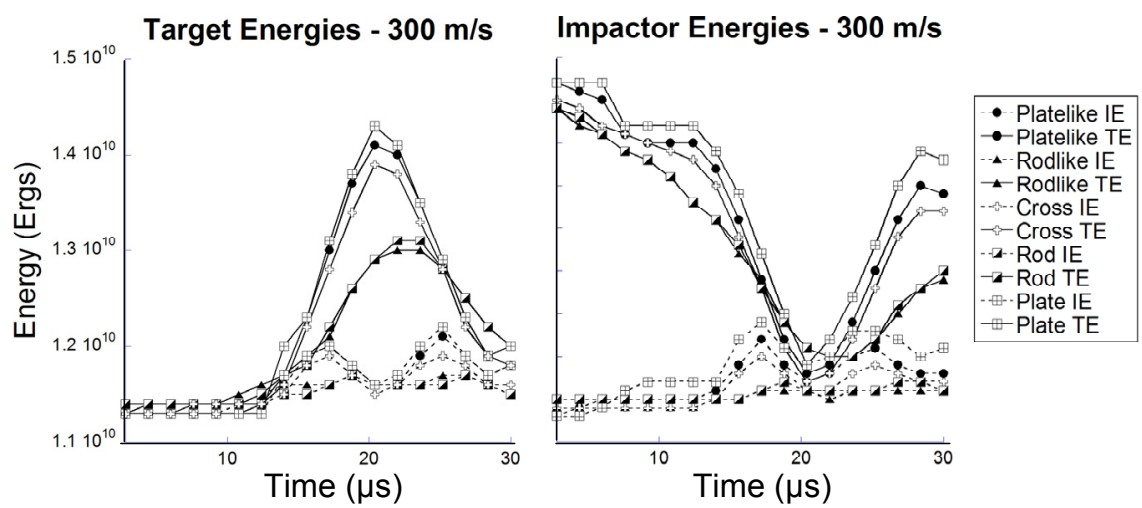

Figure 8: Internal, kinetic and total energies of the impactor and the target for the five alternative energy absorbing elements.

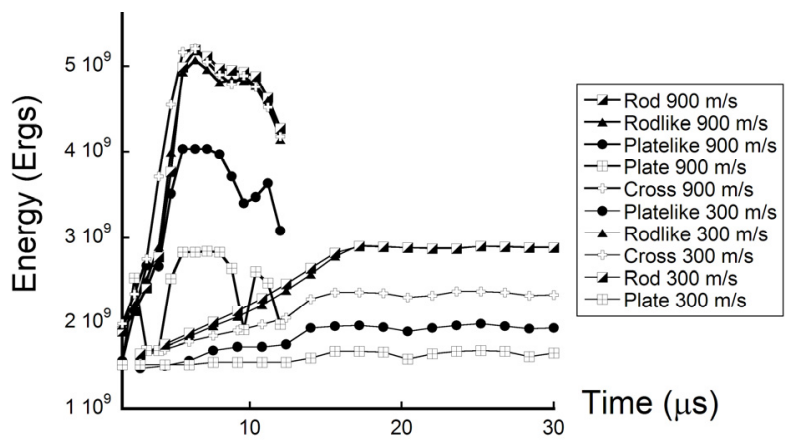

Figure 9: Internal energy generated in the EAEs for impact velocities of 300 and $900 \mathrm{~m} / \mathrm{s}$. Note that the behaviour of the cross structure, relative to the other options, depends on velocity. 
It has been found that the relative energy absorbing potential of the different EAEs depend on the impact velocity. Figure 9 shows for comparison the internal energy in the EAE vs. time curves for 300 and $900 \mathrm{~m} / \mathrm{s}$. It is evident that at $300 \mathrm{~m} / \mathrm{s}$ the cross structure lies about halfway between the plate-like and rod-like structures. But at $900 \mathrm{~m} / \mathrm{s}$ there is little difference between the cross and the rod and rod-like structures.

The differing effectiveness of the cross structure as the impact velocity changes may be explained by comparing the deformation mechanisms of the cross and rod-like structures at 300 and $900 \mathrm{~m} / \mathrm{s}$. The sequences at the top of fig. 10 show that, at low impact velocity, the voids in the cross structure close without significant lateral flow or obvious micro-jetting. By contrast, at $300 \mathrm{~m} / \mathrm{s}$ (shown at the top right of the figure), the voids in the rod-like structure fill by lateral flow. It follows that significantly more plastic work has to be done to densify the rod-like structure than the cross structure. Therefore significantly more energy is generated by the rod-like than by the cross structure. However, at $900 \mathrm{~m} / \mathrm{s}$ additional deformation mechanisms come into prominence in both the cross and rod-like structures. Grey scale plots showing plastic strain in the EAE are shown in fig. 11. As indicated, in the cross structure concentrations of flow develop between the impactor and the diagonal struts and between the diagonal struts themselves. Particularly prominent, is a "white-contrast" mushroom feature located near the original centre of the cross, which we believe is formed by penetration of the cross by a jet of impactor material. These concentrations of
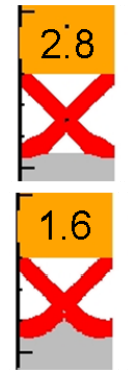
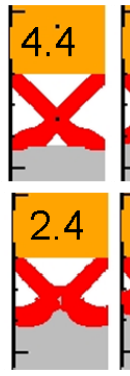
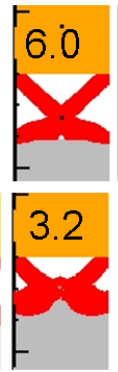
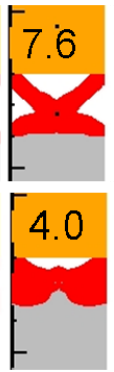
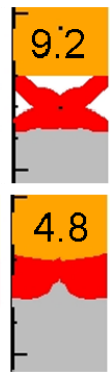
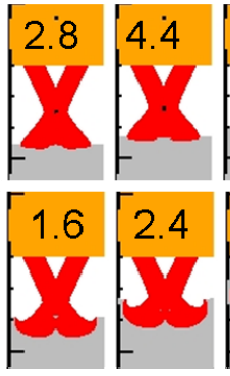
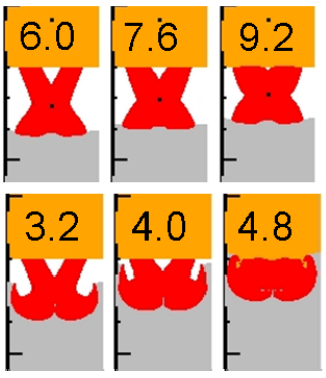

Figure 10: The top row shows cross vs. rod-like structures at $300 \mathrm{~m} / \mathrm{s}$ and the bottom row shows a similar comparison at $900 \mathrm{~m} / \mathrm{s}$.
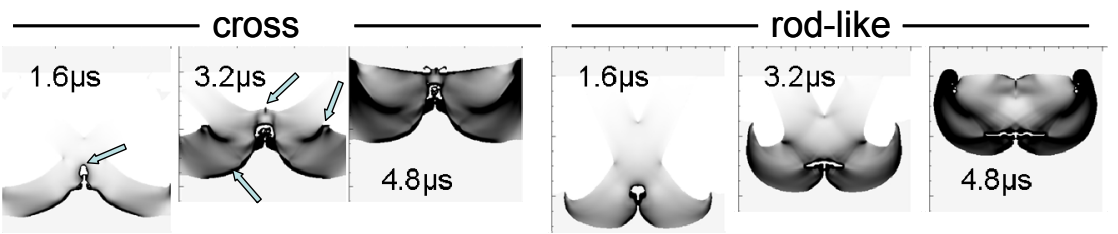

Figure 11: Plastic strain plots for cross and rod-like structures impacted at 900 $\mathrm{m} / \mathrm{s}$. Typical strain concentrations are indicated by arrows. 
deformation, or micro-kinetic flows, which are not observed when the cross variant is impacted at $300 \mathrm{~m} / \mathrm{s}$ are the probable reason for the much-increased energy absorption as velocity increases. The predicted behaviour of the rod-like structure at $900 \mathrm{~m} / \mathrm{s}$ is shown on the right of fig. 11. As with the cross, the impactor delivers a prominent jet to the EAE, but there is little evidence of micro-jetting between the struts. However, in behaviour that is similar to that which would be expected from a "rod" geometry, (see fig. 12), a mushroom is formed which occupies the whole width of the cell. In summary, at $900 \mathrm{~m} / \mathrm{s}$, both the cross and rod-like structure exhibit regions of intense flow. Although there are detailed differences in the nature of the flows in the two cases, the total internal energies absorbed are similar.

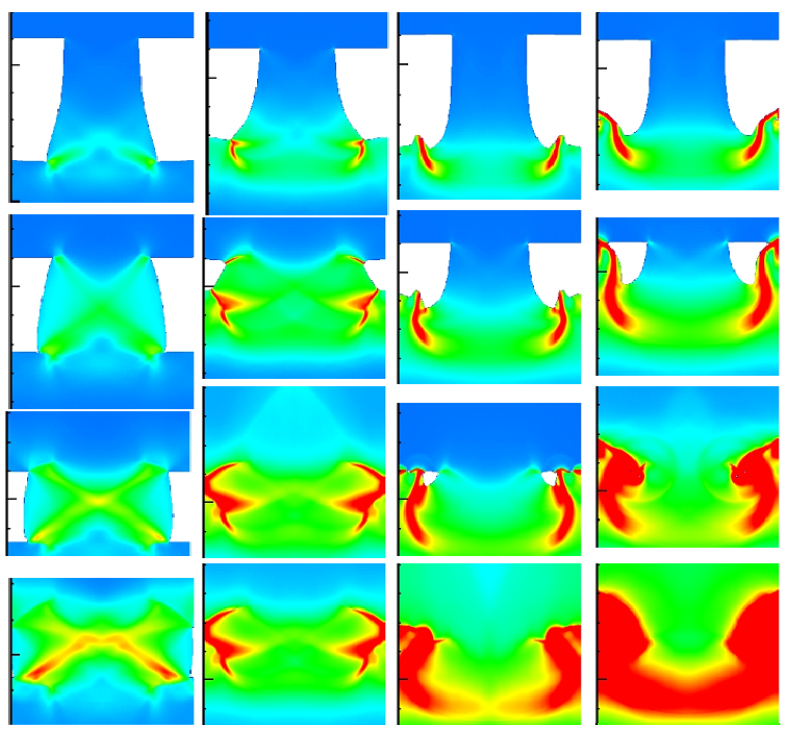

Figure 12: Internal energy plots for the rod structure. The four columns show the computed results of simulations at $300,500,700$ and $900 \mathrm{~m} / \mathrm{s}$.

This change in deformation behaviour at higher velocities to one dominated by intense plastic flow is similar to that observed by Nesterenko in studies of granular materials [7]. Nesterenko proposed that the compression of granular porous material at high rates consisted of distortions of the grains themselves combined with smaller scale deformations resulting in features such as jets and localised melting at grain boundaries. Separating the collapse process into two phases enabled the concept of micro-kinetic energy to be introduced, defined as the energy dissipated via intense plastic flow during pore collapse.

In these simulations it appears that the occurrence of the plastic flow significantly increases the energy absorbing capability of a particular structure, representing the micro-kinetic energy component of the total dissipated energy.

Figure 12 shows the results of loading a rod structure at velocities increasing from 300 to $900 \mathrm{~m} / \mathrm{s}$. The simulations clearly show that as the impact velocity is 
increased there is a transition from a mechanism dominated by roughly uniform lateral flow to one dominated by jetting. The total internal energies in the EAE are plotted in fig. 13. It is seen that the peak internal energy generated by a rod structure at $900 \mathrm{~m} / \mathrm{s}$ is nearly twice that generated at $300 \mathrm{~m} / \mathrm{s}$. We believe that the additional energy is generated by the jetting processes which come into play at higher velocities.

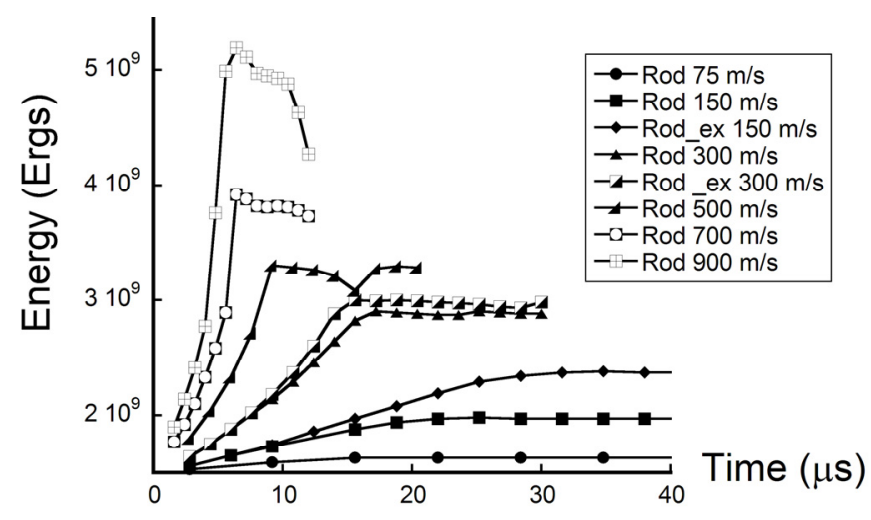

Figure 13: Total internal energy of the EAE vs. time for a rod structure impacted at different velocities. Note that at low velocities the lengths of the flyer and target become significant. Therefore, for the 150 and $300 \mathrm{~m} / \mathrm{s}$ impact, simulations were also run with the length of flyer and target extended (to $36 \mathrm{~mm}$ ). The profiles obtained are labelled here "Rod_ex $150 \mathrm{~m} / \mathrm{s}$ " and "Rod_ex $300 \mathrm{~m} / \mathrm{s}$ ".

\section{Summary and conclusions}

A cellular structure, designated MS1, has been constructed using SLM. The shock delivered to a solid target by the MS1 structure has been measured experimentally and has been calculated in 3D using CTH. CTH reproduces the main features of the experimental data but tends to underestimate the measured velocities and overestimate the amplitude of the oscillations that are superimposed on the experimental traces.

Simulations have been run on a series of cellular structures all of which occupy $40 \%$ of the volume of the cell. It was found that the internal energy generated in the cellular element increases as the structure becomes more rodlike and less plate-like. We attribute this effect to the fact that, to achieve full densification, more plastic flow takes place with the rod and rod-like structures than with the plate and plate-like structures. The relative merits of the different structures also depend on the impact velocity. Following Nesterenko and Lazaridi [8] plastic flow may be divided into the macroscopic lateral flow required to achieve full densification and jetting-type processes (micro-kinetic flow) which tend to take place at much higher strain rates than the macroscopic 
flow. A comparison between the rod-like and the cross structures was particularly informative. At $300 \mathrm{~m} / \mathrm{s}$ both structures deform in the macroscopic flow regime. However the rod-like structure has a greater capacity for lateral flow and therefore generates more internal energy than the cross structure. However at $900 \mathrm{~m} / \mathrm{s}$ the deformation of both structures tends to be dominated by micro-kinetic processes. In this regime the kinetic energies generated in the two structures are similar. A large component of micro-kinetic flow tends to be accompanied by high internal energy generation and relatively low transmission of energy across the cell.

An important aim of the single cell study was to shed light of the differences between simulation and experiment observed in the MS1 study. The MS1 structure consists of a combination of rods with different orientations relative to the impact direction. In fact MS1 could be regarded as a combination of rod and cross structures. In particular, it can be seen in fig. 10 that, as the velocity is increased from $300 \mathrm{~m} / \mathrm{s}$ to $900 \mathrm{~m} / \mathrm{s}$, the energy absorbed by the cross increases significantly more than the energy absorbed by a rod. It is possible that the actual behaviour of a cross, or more generally an angled strut, is not reproduced accurately by the code. For example, suppose the micro-kinetic flow predicted by the code did not occur to the same extent in reality. This would mean that the code was overestimating the energy absorbed, especially at high velocities. A consequence would be that, as observed experimentally, the code would underestimate the amplitude of the wave reaching the back surface of the target with the discrepancy being greater at higher velocities. Another significant difference between the experiments and the $\mathrm{CTH}$ simulations was that the amplitude of the oscillations resulting from successive pore collapse was less in the experiment than in the simulations. It is important to note that the fact that the oscillations observed are smaller that the code may be due to limited resolution in the unfolding of the experimental data. However the observation is consistent with the hypothesis, described above, that the micro-kinetic flows may be less in reality than in the CTH predictions.

In future work we will perform experiments on SLM structures which are simpler that MS1. The aim will be to determine the code's ability to reproduce the behaviour of elements such as angled struts, crosses and rods. This should enable us to better evaluate the ability of CTH to model the energy absorbing capability of cellular structures thereby supporting the development of structures manufactured by SLM, and other techniques, for use in protection against fragment, blast, and other high rate loading.

Finally, we thank Steven Johnson of the Institute of Shock Physics, Imperial College, London for experimental support.

\section{References}

[1] R. A. W. Mines, S. McKown, W. Cantwell, S. Tsopanos, W. Brooks and C. J. Sutcliffe, High performance sandwich structures with hierarchical lattice cores, ICEM13 (E. E. Gdoutos, Ed) Springer, Dordrecht, Paper No. 158 (2007). 
[2] V. S. Deshpande and N. A. Fleck, Collapse of truss core sandwich beams in 3-point bending, Int. J. Sol. Struct. 38, 6275-6305 (2001).

[3] J. Wang, A. G. Evans, K Dharmasena and H. N. G. Wadley, On the performance of truss panels with Kagome cores, Int. J. Sol. Struct. 40, 6981-6988 (2003).

[4] S. McKown, Y. Shen, W. K. Brookes, C. J. Sutcliffe, W. J. Cantwell, G. S. Langdon, G. N. Nurick and M. D. Theobald, The quasi-static and blast loading response of lattice structures, Int J Impact Eng 35, (2008) 795-810.

[5] E. S. Hertel, R. L. Bell, M. G. Elrick, A. V. Farnsworth, G. I. Kerley, J. M. McGlaun, S. V. Petney, S. A. Silling, P. A. Taylor and L. Yarrington, "CTH: A Software Family for Multi-Dimensional Shock Physics Analysis", Proc Int Conf on Shock Waves, Vol. 1, pp. 377-382, Marseille, France, July, 1993.

[6] D. J. Steinberg, S. G. Cochran, and M. W. Guinan, (1980), A Constitutive model for metals applicable at high strain rates, J Appl Phys. 51 1498-1504.

[7] O. T. Strand, D. R. Goosman, C. Martinez, T. L. Whitworth and W. W. Kuhlow, Compact system for high-speed velocimetry using heterodyne techniques, Rev. Sci. Instrum. 77, 083018 (2006).

[8] V. F. Nesterenko and A. N. Lazaridi, Regimes of shock-wave compaction of granular materials, High Pressure Science and Technology: Proceedings of XII AIRAPT and XXVII EMPRG International Conference, ed. W. B. Holzapfel and P. G. Johnson, 835-837, Gordon and Breach, New York (1990).

(C) British Crown Owned Copyright 2012 /AWE. Published with the permission of the Controller of Her Britannic Majesty's Stationery Office. 